\title{
The Association Study of PRKG1 Gene Polymorphism and Salt-Sensitive Hypertension among the Essential Hypertension in Beijing
}

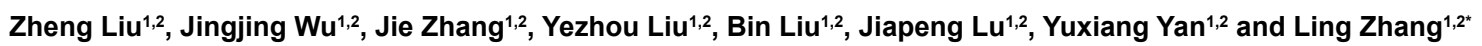

${ }^{1}$ Department of Epidemiology and Biostatistics, School of Public Health, Capital Medical University, Beijing, 100069, People's Republic of China ${ }^{2}$ Beijing Municipal Key Laboratory of Clinical Epidemiology, Beijing, People's Republic of China

\begin{abstract}
Background: PRKG1 plays an important role in regulating vascular smooth muscle contraction. And this gene is regarded as one of the candidate genes on salt-sensitive hypertension. The aim of this study was to investigate the effects of environmental risk factors and two SNPs and haplotypes structures of PRKG1 gene with blood salt-sensitive patients among essential hypertension.

Method: Three hundred and forty-two essential hypertensive were recruited in a case-case study, a modified Sullivan's acute salt load method was conducted to identify the salt-sensitivity and salt-resistant. Medical history and lifestyle risk factors were obtained by questionnaire, and blood and urine specimens were collected to test the biochemical indicators. We used the Sequenom Mass ARRAY Platform for genotyping of $P R K G 1$ gene single nucleotide polymorphisms (SNPs) (rs7897633/SNP1, rs1904694/SNP2). We applied statistical software SPSS17.0, multifactor dimensionality reduction method 1.1 .0 and haploview 4.0 to do the analyses.
\end{abstract}

Results: There were 63 salt sensitivity subjects in 342 essential hypertension individuals, accounting for $18.4 \%$ of the total. Age and 24-hour urinary sodium concentration were the risk factors associated with salt-sensitive hypertension. The results of the logistic regression model showed that subjects carrying SNP1-AA genotype and SNP2-GG genotypes had 2.83 -fold $(95 \% \mathrm{Cl}: 1.21-6.63)$ and 3.50 -fold $(95 \% \mathrm{Cl}: 1.54-7.93)$ risks to have salt-sensitive hypertension, respectively. Haplotype analysis showed that haplotype CA had 0.56-fold (95\% Cl: $0.38-0.84)$ decreased risk for salt-sensitive hypertension, whereas, haplotype $A G$ had 1.78 -fold $(95 \% \mathrm{Cl}: 1.20-2.65)$ increased risk for saltsensitive hypertension.

Conclusion: Age and 24-hour urinary sodium concentration are the environmental risk factors associated with salt-sensitive hypertension. Genotypes of SNP1-AA, SNP2-GG and haplotype AG of PRKG1 gene were risk factors for salt-sensitivity in essential hypertension.

Keywords: Salt sensitivity; Essential hypertension; Age; 24-hour urinary sodium concentration; PRKG1 gene; Single nucleotide polymorphism; Haplotype

\section{Introduction}

Essential hypertension is one of the most common cardiovascular diseases that are seriously harmful to human health, and it is influenced by genetic and environmental factors [1]. High salt intake is the most important environmental risk factor. Salt-sensitive hypertension can be regarded as a subtype of essential hypertension; however, it is very heterogeneous among individuals. In China, a research study suggested that there is nearly sixty percent of essential hypertensive with saltsensitivity, while the general population is at twenty-five percent [2]. The characteristic of salt-sensitive hypertension significantly increases blood pressure after a salt loading test or decreases after a urinary sodium excretion test. This means that salt load is positively related with elevation of blood pressure [3]. Thus, it is possible to decrease the cardiovascular morbidity and mortality relatively by reducing dietary salt intake. In recent years, genetic biomarkers of salt-sensitivity have been taken notice by many researchers. Some studies indicate that many pathogenic mechanisms may cause salt-sensitive hypertension, such as the sympathetic nervous system, the Renin-AngiotensinAldosterone System (RAAS) and endothelial functional dysregulation $[4,5]$. In these pathways, researchers have already found some Single Nucleotide Polymorphism (SNP) loci, which are associated with saltsensitivity in the candidate genes studies [6-10]. Several researches show that $P R K G 1$ protein ( $c G M P$-dependent protein kinase 1) can regulate vascular smooth muscle contraction by reducing the biological activity of nitric oxide or relatively increasing intracellular $\mathrm{Ca}^{2+}$ concentration
$[11,12]$ to increase blood pressure. Further study suggests that a SNP locates in the first introns of PRKG1 gene (rs7897633) is associated with variation in diastolic blood pressure after acute salt load [13] In this study we discuss the association between PRKG1 gene single nucleotide polymorphism (rs7897633, rs1904694) and salt-sensitive hypertension among essential hypertensive in Beijing, and investigating the interaction of the two SNPs as well as the effect of haplotypes on salt-sensitive hypertension.

\section{Materials and Methods}

\section{Subjects}

Three hundred and forty-two essential hypertensive were recruited in a case-case study in Beijing, a modified Sullivan's acute salt load method [14] was conducted to identify the Salt-Sensitive Hypertension (SSH) and Salt-Resistant Hypertension (SRH) Subjects who had

*Corresponding author: Ling Zhang, School of Public Health, Capital Medical University. No.129 mail box, No.10 Xitoutiao, Youanmenwai, Beijing, 100069, the People's Republic of China, Tel/Fax: 861083911777 ; E-mail: zlilyepi@ccmu.edu.cn

Received June 13, 2014; Accepted July 23, 2014; Published July 27, 2014

Citation: Liu Z, Wu J, Zhang J, Liu Y, Liu B, et al. (2014) The Association Study of PRKG1 Gene Polymorphism and Salt-Sensitive Hypertension among the Essential Hypertension in Beijing. J Hypertens 3: 162. doi:10.4172/21671095.1000162

Copyright: @ 2014 Liu Z, et al. This is an open-access article distributed under the terms of the Creative Commons Attribution License, which permits unrestricted use, distribution, and reproduction in any medium, provided the original author and source are credited. 
one of these criteria would be involved in the study: 1) No taking of antihypertensive drugs with Blood Pressure (BP) measurement three times in different days, systolic blood pressure $(\mathrm{SBP}) \geq 140$ $\mathrm{mmHg}$ and/or diastolic blood pressure (DBP) $\geq 90 \mathrm{mmHg}$ or taking antihypertensive drugs; 2) Have a history of hypertension and use antihypertensive drugs currently, with BP is lower than $140 / 90 \mathrm{mmHg}$; 3) Diagnosed with essential hypertension by hospitals of level two or above. We excluded from this study, subjects with cardiomyopathy, valvular heart disease, congenital heart disease, acute myocardial infarction, stroke, type 2 diabetes, and secondary hypertension.

The study was approved by Ethical Committee, Capital Medical University, Beijing, China. All participants were given informed consent before participating.

\section{Measurement of anthropometric parameters}

The questionnaire covered general condition, family history, physical examination, personal behavior, and medical history. Body weight, height, waist circumference, hip circumference, baseline systolic blood pressure and diastolic blood pressure were measured by well-trained community doctors. Each measurement was performed three times and the average value was calculated as a final reading. Then we calculated mean arterial pressure (MAP) by SBP and DBP value $\left(\mathrm{MAP}=\left(\mathrm{SBP}+2^{*} \mathrm{DBP}\right) / 3\right)$. Peripheral venous blood was collected in the morning after an overnight fasting for the blood biochemical examination, such as blood glucose, total cholesterol, triglyceride, high-density lipoprotein cholesterol and low-density lipoprotein cholesterol, etc. At the same time, food frequency tables and twentyfour hours urinary sodium excretion were used to evaluate the daily sodium intake of individuals. A modified Sullivan's acute salt load method was conducted to identify the salt-sensitivity and salt-resistant. Blood pressure was measured by mercury sphygmomanometer on the right arm of the participant in a comfortable sitting position after at least a 5 minutes rest. Blood pressure and time-point urinary electrolyte concentration were measured after acute salt load and furosemide natriuretic test for two hours. Anticoagulant blood samples were centrifuged, and then stored at $-80^{\circ} \mathrm{C}$ instantly.

\section{Phenotype and Genotype}

Magnetic beads method for genomic DNA extraction kit (BioTeke biological technology co., LTD., Beijing, AU1101) was used to extract genome DNA in peripheral blood leukocytes. We used Sequenom Mass ARRAY Platform (San Diego, Calif) for genotyping of PRKG1 gene SNPs (SNP1: rs7897633; SNP2: rs1904694). The main steps of experiments are as follows:

1. Using the Assay design 3.1 (Sequenom, San Diego, California, SA) for designing primers.

2. Polymerase chain reaction (PCR) plate with 384 holes was applied for amplifying high-throughput multiple PCR to the target fragment

3. SAP nitration reaction

4. Single-base extension

5. Using the resin for desalination and purification after the reaction products were diluted for three times

6. After desalting, the samples were added on the target, until natural crystals formed. Afterwards, mass spectrometry and data was collected.

\section{Statistical Analysis}

The statistical analyses were carried out using SPSS version 17.0 for Windows (SPSS Inc., Chicago, IL, USA). For continuous variables with normal distributions, we used two independent sample t-test and ANOVA to detect the environmental factors between SSH and SRH groups and the difference of distribution between the different genotypes, respectively. For variables with non-normal distributions, we applied the Rank Sum Test. Each polymorphism was evaluated for Hardy-Weinberg equilibrium by $\chi^{2}$ test. $\mathrm{P} \geq 0.05$ was considered to obey the Hardy-Weinberg equilibrium. The distributions of allelic and genotypic frequencies were analyzed by $\chi^{2}$ test. The single locus association between a polymorphism and salt-sensitive hypertension was analyzed by multiple logistic regression analysis. The interactions were associated with salt sensitivity between the tag-SNPs were analyzed by multifactor dimensionality reduction method (MDR) (version 1.1.0; Computational Genetics Laboratory, Dartmouth Medical School, Lebanon, NH; www.epistasis.org). The frequencies of the haplotypes and association analysis were completed by Haploview software (version 4.0; Mark Daly's Laboratory, Broad Institute; http:// sourceforge.net/projects/haploview/). All probability values were for two-tailed tests and $\mathrm{P}<0.05$ was considered to be significant statistically.

\section{Results}

\section{Environmental factors analysis for salt-sensitive hypertension}

The characteristics of the subjects are shown in Table 1 . There were 63 salt sensitivity subjects in 342 essential hypertensive, accounting for $18.4 \%$ of the total. There were $19.7 \%$ and $15.3 \%$ SSH in female and male, respectively, no statistically significant difference was detected. The average age of all the participants was $57.61 \pm 8.50$ years old, and the average age of SSH group was significant higher than SRH group ( $59.62 \pm 8.95$ vs. $57.16 \pm 8.35$ years old, $P=0.038$ ). The proportion of SSH increased progressively with age $(11.9 \%, 18.0 \%, 20.1 \%$ and $30.4 \%$ in 40-, 50-, 60- and 70- years old). Twenty-four hours urinary sodium concentration was the risk factor associated with salt sensitivity, while there were no significant differences between the two groups in height, weight, waist circumference, hip circumference, BMI level, gender, smoking and drinking status, family history of hypertension, baseline blood pressure, biochemical indicators and twenty-four hours urinary potassium concentration (Table 1).

\section{Different genotypes of $P R K G 1$ gene and blood pressure variation analysis}

We used ANOVA to detect the difference of blood pressure variation between the different genotypes after salt load and furosemide natriuretic test for two hours. The results were show $\triangle \mathrm{MABP} \mathrm{P}_{1}$ when compared to those with the AA genotype between two groups after salt load for two hours of SNP1. Moreover, the subjects who carried CA or AA genotype changed bigger at $\triangle \mathrm{MABP}_{2}$ than those with the $\mathrm{CC}$ genotype between two groups after furosemide natriuretic test for two hours of SNP1. No significant differences were found in mean arterial pressure between different genotypes.

We also observed that AA genotype carriers had a big change at $\triangle \mathrm{MABP}_{1}$ after salt load for two hours of SNP2 in SS group. The subjects who carried GG or GA genotype changed bigger at $\triangle \mathrm{MABP}_{2}$ when compared with the AA genotype carriers between two groups after furosemide natriuretic test for two hours of SNP2. No significant differences were found in mean arterial pressure between different genotypes. 
Citation: Liu Z, Wu J, Zhang J, Liu Y, Liu B, et al. (2014) The Association Study of PRKG1 Gene Polymorphism and Salt-Sensitive Hypertension among the Essential Hypertension in Beijing. J Hypertens 3: 162. doi:10.4172/2167-1095.1000162

Page 3 of 7

\begin{tabular}{|c|c|c|c|c|c|}
\hline \multicolumn{2}{|c|}{ Characteristics } & Total & SSH & SRH & $P$ \\
\hline \multicolumn{2}{|c|}{ Number (\%) } & 342 & $63(18.4)$ & 279 (81.6) & - \\
\hline \multicolumn{2}{|c|}{ Gender (Woman, N, \%) } & $238(69.6)$ & $47(74.6)$ & $191(68.5)$ & $0.286^{\#}$ \\
\hline \multicolumn{2}{|c|}{ Age (year) } & $57.61 \pm 8.50$ & $59.62 \pm 8.95$ & $57.16 \pm 8.35$ & $0.038^{\Delta}$ \\
\hline \multicolumn{2}{|c|}{ Height $(\mathrm{cm})$} & $159.24 \pm 7.80$ & $158.50 \pm 7.36$ & $159.41 \pm 7.90$ & $0.408^{\Lambda}$ \\
\hline \multicolumn{2}{|c|}{ Weight (kg) } & $69.94 \pm 11.50$ & $69.16 \pm 11.44$ & $70.11 \pm 11.53$ & $0.555^{\wedge}$ \\
\hline \multicolumn{2}{|c|}{ BMI $\left(\mathrm{kg} / \mathrm{m}^{2}\right)$} & $27.51 \pm 3.62$ & $27.47 \pm 3.61$ & $27.52 \pm 3.62$ & $0.916^{\wedge}$ \\
\hline \multicolumn{2}{|c|}{$W C(\mathrm{~cm})$} & $90.82 \pm 8.95$ & $91.27 \pm 9.42$ & $90.72 \pm 8.85$ & $0.658^{\wedge}$ \\
\hline \multicolumn{2}{|c|}{$\mathrm{HC}(\mathrm{cm})$} & $101.36 \pm 7.20$ & $102.11 \pm 8.68$ & $101.19 \pm 6.83$ & $0.360^{\Delta}$ \\
\hline \multicolumn{2}{|c|}{ waist-to-hip ratio } & $0.90 \pm 0.06$ & $0.89 \pm 0.05$ & $0.89 \pm 0.06$ & $0.761^{\perp}$ \\
\hline \multicolumn{2}{|c|}{ FBG (mmol/L) } & $5.91 \pm 1.44$ & $5.96 \pm 1.55$ & $5.89 \pm 1.42$ & $0.730^{8}$ \\
\hline \multicolumn{2}{|c|}{ TG (mmol/L) } & $1.96 \pm 1.42$ & $2.10 \pm 1.73$ & $1.92 \pm 1.34$ & $0.351^{8}$ \\
\hline \multicolumn{2}{|c|}{$\mathrm{TC}(\mathrm{mmol} / \mathrm{L})$} & $5.03 \pm 1.00$ & $5.16 \pm 0.88$ & $5.00 \pm 1.02$ & $0.239^{\wedge}$ \\
\hline \multicolumn{2}{|c|}{$\mathrm{HDL}(\mathrm{mmol} / \mathrm{L})$} & $1.42 \pm 0.34$ & $1.43 \pm 0.31$ & $1.42 \pm 0.35$ & $0.851^{\wedge}$ \\
\hline \multicolumn{2}{|c|}{$\mathrm{LDL}(\mathrm{mmol} / \mathrm{L})$} & $3.48 \pm 0.34$ & $3.76 \pm 1.05$ & $3.37 \pm 1.05$ & $0.118^{\wedge}$ \\
\hline \multicolumn{2}{|c|}{$\operatorname{ALT}(\mu / L)$} & $17.59 \pm 13.82$ & $19.38 \pm 10.61$ & $17.25 \pm 14.36$ & $0.187^{8}$ \\
\hline \multicolumn{2}{|c|}{ AST $(\mu / L)$} & $17.65 \pm 5.96$ & $19.36 \pm 7.58$ & $17.32 \pm 5.58$ & $0.169^{8}$ \\
\hline \multicolumn{2}{|c|}{$\mathrm{SC}(\mu \mathrm{mol} / \mathrm{L})$} & $51.02 \pm 10.25$ & $51.86 \pm 11.75$ & $50.59 \pm 9.60$ & $0.713^{\Delta}$ \\
\hline \multicolumn{2}{|c|}{$\mathrm{UA}(\mu \mathrm{mol} / \mathrm{L})$} & $334.49 \pm 89.39$ & $338.21 \pm 127.75$ & $332.56 \pm 64.20$ & $0.850^{\wedge}$ \\
\hline \multicolumn{2}{|c|}{ High salt diet $\mathrm{N}(\%)$} & $21(30.9)$ & $7(31.8)$ & $14(30.4)$ & $0.908^{\#}$ \\
\hline \multicolumn{2}{|c|}{$\mathrm{FH}$ of hypertension $\mathrm{N}(\%)$} & $192(56.1)$ & $28(80.0)$ & $164(74.2)$ & $0.462^{\#}$ \\
\hline \multicolumn{2}{|c|}{$\mathrm{SBP}(\mathrm{mmHg})$} & $142.23 \pm 17.19$ & $142.97 \pm 18.68$ & $142.06 \pm 16.86$ & $0.707^{\perp}$ \\
\hline \multicolumn{2}{|c|}{$\mathrm{DBP}(\mathrm{mmHg})$} & $85.28 \pm 10.29$ & $85.36 \pm 10.84$ & $85.26 \pm 10.18$ & $0.945^{\wedge}$ \\
\hline \multicolumn{2}{|c|}{$\operatorname{MAP}(\mathrm{mmHg})$} & $104.26 \pm 10.70$ & $104.56 \pm 11.19$ & $104.20 \pm 10.60$ & $0.806^{\wedge}$ \\
\hline \multicolumn{2}{|c|}{ HR (time/min) } & $73.82 \pm 11.33$ & $75.80 \pm 11.65$ & $73.47 \pm 11.28$ & $0.300^{\Delta}$ \\
\hline \multicolumn{2}{|c|}{ Concentration of $24 \mathrm{hNNa}$} & $115.55 \pm 49.18$ & $104.03 \pm 45.95$ & $118.14 \pm 49.59$ & $0.045^{\star}$ \\
\hline Conce & on of $24 \mathrm{hNK}$ & $29.05 \pm 12.68$ & $28.27 \pm 11.75$ & $29.23 \pm 12.89$ & $0.598^{\wedge}$ \\
\hline Con & f $24 \mathrm{hNNa}$ & $193.64 \pm 90.94$ & $179.47 \pm 87.59$ & $196.84 \pm 91.53$ & $0.182^{8}$ \\
\hline & of $24 \mathrm{hNK}$ & $48.11 \pm 21.29$ & $47.98 \pm 19.58$ & $48.13 \pm 21.70$ & $0.878^{8}$ \\
\hline The status of Me & on today $(\mathrm{N}, \%, \mathrm{n}=261)$ & $220(84.3)$ & $32(84.2)$ & $188(84.3)$ & $0.988^{\#}$ \\
\hline Medication regularly & Regularly & $53(77.9)$ & $17(77.3)$ & $36(78.3)$ & $0.821^{8}$ \\
\hline & Irregularly & $9(13.2)$ & $2(9.1)$ & $7(15.2)$ & \\
\hline$(\%, n=68)$ & Don't take medicine & $6(8.8)$ & $3(4.4)$ & $3(6.5)$ & \\
\hline Smoking & Everyday & $64(19.5)$ & $13(22.0)$ & $51(19.0)$ & $0.410^{8}$ \\
\hline & Not every day or never & $239(72.9)$ & $43(72.9)$ & $196(72.9)$ & \\
\hline$(\%, n=328)$ & Quit smoking & $25(7.6)$ & $3(5.1)$ & $22(8.2)$ & \\
\hline Drinking & Almost once a day & $40(12.4)$ & $8(13.5)$ & $32(12.2)$ & $0.918^{8}$ \\
\hline & 3-4 times/week & $7(2.2)$ & $0(0)$ & $7(2.6)$ & \\
\hline$(\%, n=322)$ & 1-2 times/week & $17(5.3)$ & $5(8.5)$ & $12(4.6)$ & \\
\hline & 1-3 times/mouth & $17(5.3)$ & $2(3.4)$ & $15(5.7)$ & \\
\hline & Almost never & $241(74.8)$ & $44(74.6)$ & $197(74.9)$ & \\
\hline
\end{tabular}

Note: Values are mean \pm SD or number and percentage. $P$ values are calculated by $X^{2}\left({ }^{\#}\right)$ or $T$ test $\left({ }^{\Delta}\right)$ or rank sum test $\left({ }^{\circledR}\right)$. ${ }^{*} \mathrm{P}<0.05$. concentration of $24 \mathrm{hNNa:}$ Twenty-Fou Hours Urine Sodium Concentration; concentration of 24 hNK: Twenty-Four Hours Urinary Potassium Concentration; content of 24hNNa: Twenty-Four Hours Urine Sodium Content; content of 24 hNK: Twenty-Four Hours Urinary Potassium Content; FH of hypertension: Family History of Hypertension; SSH: Salt-Sensitivity Hypertension; SRH: Salt-Resistance Hypertension; BMI: Body Mass Index; WC: Waist Circumference; HC: Hip Circumference; FBG: Fasting Blood Glucose; TG: Triglyceride; TC: Total Cholesterol; HDL: high density lipoprotein; LDL: low density lipoprotein; ALT: Glutamic-Pyruvic Transaminase; AST: Glutamic Oxalacetic Transaminase; SC: Serum Creatinine; UA: Uric Acid; SBP: Systolic Blood Pressure; DBP: Diastolic Blood Pressure; MAP: Mean Artery Blood Pressure; HR: Heart Rate Table 1: Baseline characteristics of study participant by salt-sensitivity and salt-resistance.

\section{Association between genotypic and allelic frequencies distributions with salt-sensitive hypertension}

Analysis showed that the distributions of genotypic frequencies were in Hardy-Weinberg equilibrium at the two tag-SNPs (SNP1: SSH group $P_{H W E}=0.866$, SRH group $P_{H W E}=0.973$, SNP2: SSH group $P_{H W E}=0.701$, SRH group $\left.P_{H W E}=0.477\right)$, suggesting that the genotypes samples of this study could be inherited stably. Table 2 showed the distribution of genotypes and alleles frequencies of SNP1 and SNP2 on essential hypertension, and there were statistically significant differences between the two tag-SNPs in the SS and SR groups (SNP1:
$P=0.041$, SNP2: $P=0.007)$. Multiple logistic regression analysis was used to discuss the associations between SNP1 and SNP2 with salt-sensitive hypertension, respectively. The analysis (age, twenty-four hours urinary sodium concentration and the two tag-SNPs adjusted) identified that participants with the SNP1-AA genotype were 2.83-fold (95\% CI: 1.216.63) at risk when compared to those with the SNP1-CC genotype, and those with the SNP2-GG genotype were 3.50-fold (95\% CI: 1.54 7.93) at risk when compared to those with the SNP2-AA genotype for salt-sensitive hypertension. Meanwhile, Chi-square analysis results revealed that participants with the SNP1-A allele were 1.66-fold (95\% CI: 1.12-2.47) at risk for salt-sensitive hypertension when compared to 
Citation: Liu Z, Wu J, Zhang J, Liu Y, Liu B, et al. (2014) The Association Study of PRKG1 Gene Polymorphism and Salt-Sensitive Hypertension among the Essential Hypertension in Beijing. J Hypertens 3: 162. doi:10.4172/2167-1095.1000162

Page 4 of 7

\begin{tabular}{|c|c|c|c|c|c|c|c|}
\hline SNPs & Polymorphism & SSH & SRH & $x^{2}$ & $P^{*}$ & OR & $95 \% \mathrm{Cl}$ \\
\hline SNP1 & Genotype & & & & & & \\
\hline & AA & $23(37.10)$ & $63(23.08)$ & \multirow[t]{3}{*}{6.39} & \multirow[t]{3}{*}{$0.041^{*}$} & 2.83 & $1.21-6.63$ \\
\hline & $A C$ & $29(46.77)$ & $136(49.82)$ & & & 1.52 & $0.67-3.43$ \\
\hline & CC & $10(16.13)$ & 74 (27.11) & & & 1.00 & - \\
\hline & Allele & & & & & & \\
\hline & A & $75(60.48)$ & 262 (47.99) & \multirow[t]{2}{*}{6.31} & \multirow[t]{2}{*}{$0.012^{*}$} & \multirow[t]{2}{*}{1.66} & \multirow[t]{2}{*}{$1.12-2.47$} \\
\hline & C & $49(39.52)$ & $284(52.01)$ & & & & \\
\hline SNP2 & Genotype & & & & & & \\
\hline & GG & $16(26.23)$ & $32(11.68)$ & \multirow[t]{3}{*}{10.06} & \multirow[t]{3}{*}{$0.007^{\circ}$} & 3.50 & $1.54-7.93$ \\
\hline & GA & $29(47.54)$ & $131(47.81)$ & & & 1.49 & $0.75-2.96$ \\
\hline & AA & $16(26.23)$ & $111(40.51)$ & & & 1.00 & - \\
\hline & Allele & & & & & & \\
\hline & G & $61(50.00)$ & 195 (35.58) & \multirow[t]{2}{*}{8.78} & \multirow[t]{2}{*}{$0.003^{*}$} & \multirow[t]{2}{*}{1.81} & \multirow[t]{2}{*}{$1.22-2.69$} \\
\hline & A & $61(50.00)$ & $353(64.42)$ & & & & \\
\hline
\end{tabular}

Note: $\mathrm{n}(\%)$, numbers and frequencies; ${ }^{*} P<0.05$, P values were calculated by $X^{2}$ test; OR, odd ratio; $95 \% \mathrm{Cl}, 95 \%$ confidence interval; OR was calculated by multiple logistic regression adjusted by age, twenty-four hours urinary sodium concentration and the two tag-SNPs

Table 2: The genotypes and allele frequencies of PRKG1 SNPs in salt-sensitive hypertension and salt-resistant hypertension.
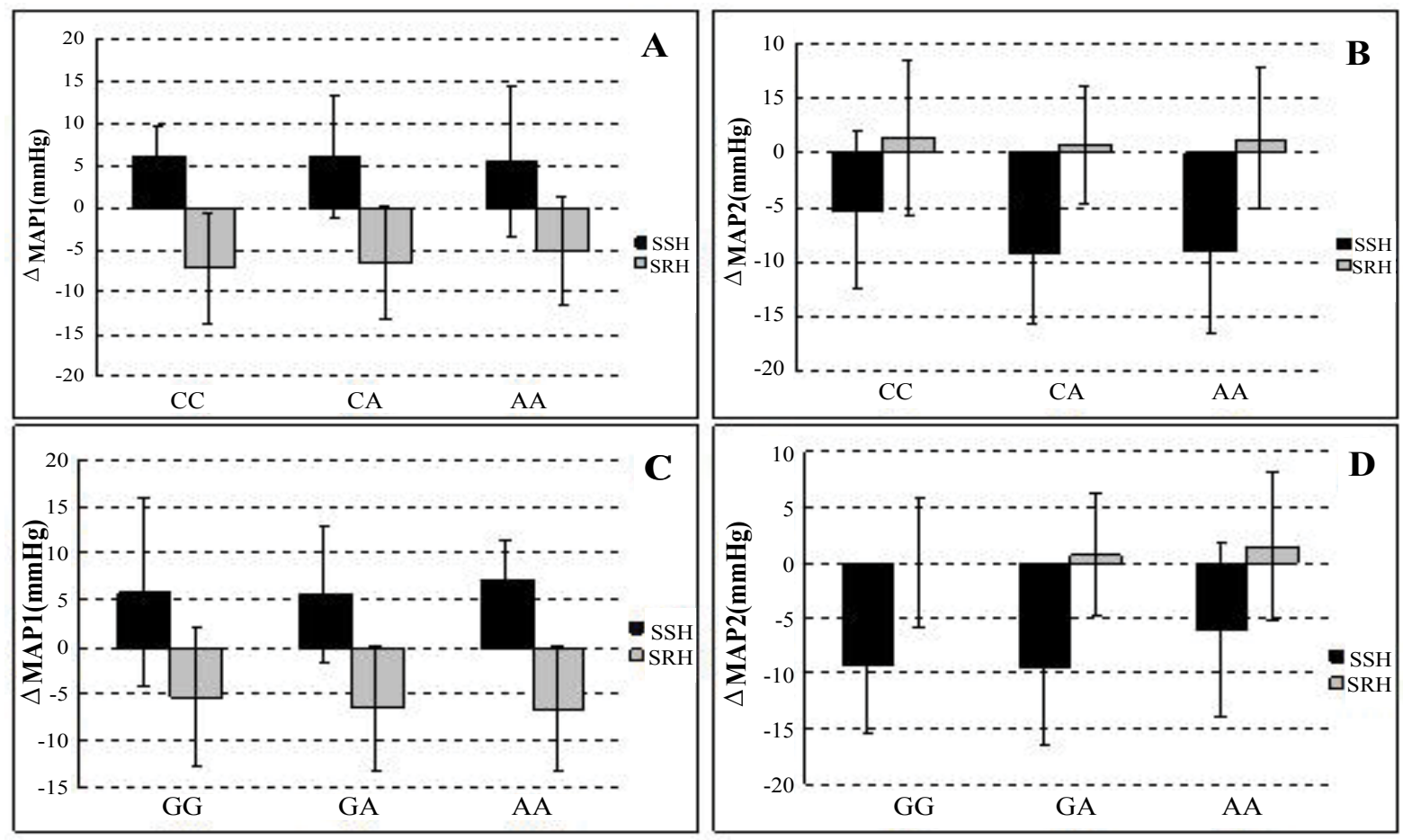

Note: A. $\triangle M A P_{1}$ variation of SNP1 after salt load for two hours; B. $\triangle M^{\prime} P_{2}$ variation of SNP1 after furosemidum test for two hours; C. $\triangle M A P_{1}$ cariation of SNP2 after salt load For two hours; D. $\triangle \mathrm{MAP}_{2}$ variation of SNP2 after furosemidum test for two hours

Figure 1: Different genotypes of PRKG1 gene and blood pressure variation.

those with the SNP1-G allele. And SNP2-G allele were 1.81-fold (95\% CI: 1.22-2.69) at risk for salt-sensitive hypertension when compared to those with SNP2-A allele.

Interaction analysis of PRKG1 gene tag-SNPs and environmental factors on salt-sensitive hypertension

MDR analysis was used to detect the interaction between each of the two tag-SNPs (SNP1 and SNP2) and twenty-four hours urinary sodium content. Table 3 summarized the best interaction models. In one-factor model, SNP1 was the best attribute for predicting saltsensitive hypertension (testing accuracy $=52.21 \%$; $C V C=6 / 10, P=0.377$ ).

\begin{tabular}{|c|c|c|c|c|}
\hline Model & $\begin{array}{c}\text { Training } \\
\text { Bal. Acc }\end{array}$ & $\begin{array}{c}\text { Testing Bal. } \\
\text { Acc }\end{array}$ & Sign test (P) & $\begin{array}{c}\text { Cross-Validation } \\
\text { Consistency }\end{array}$ \\
\hline SNP1 & 0.5858 & 0.5221 & $6(0.377)$ & $6 / 10$ \\
\hline 24hNNa+SNP1 & 0.6151 & 0.6151 & $8(0.055)$ & $10 / 10$ \\
\hline $\begin{array}{c}24 \mathrm{hNNa} \\
\text { +SNP1+SNP2 }\end{array}$ & 0.6430 & 0.6067 & $9\left(0.011^{\star}\right)$ & $10 / 10$ \\
\hline
\end{tabular}

Note: * $\mathrm{P}<0.05$

Table 3: Best models of interaction analysis of PRKG1 gene-environment factors.

Twenty-four hours urinary sodium content and SNP1 was the best two-factor model (testing accuracy=61.51\%, CVC=10/10, $P=0.055$ ). But by following the best model selected principle, the best model was 
determined to be a gene-environmental model, which included the polymorphisms of SNP1, SNP2 and twenty-four hours urinary sodium content, with a better testing balance accuracy to $60.67 \%$ and a perfect Cross-Validation Consistency of $10 / 10(P=0.011)$. Thus, the interaction dendrogram (Figures 1 and 2) showed that there was no significant difference between the two tag-SNPs linked by blue lines. Furthermore, the dendrogram also indicated that there were moderate strength interactions between the two tag-SNPs with twenty-four hours urinary sodium content associated with salt-sensitivity.

\section{Haplotypes analysis of the two tag-SNPs of PRKG1 gene}

Haploview 4.0 software was applied to analyze the frequency of haplotype, and there was a strong linkage disequilibrium between the two SNPs $\left(D^{\prime}=0.838, P<10^{-4}\right)$. As it was showed in Table 4 , four possible haplotypes were derived from the observed genotypes. Three haplotypes with frequencies above $5 \%$ were Haplotype1 (H1)-CA (46.9\%), Haplotype 2 (H2)-AG (34.8\%) and Haplotype3 (H3)-AA (15.2\%). The results revealed that there were significant frequency differences between SS and SR groups in H1 (SNP1-C/SNP2-A) and H2 (SNP1-A/SNP2-G). H1 was more common in the SR group (49.47\%) compared to the SS $(35.81 \%, P=0.006)$, while $\mathrm{H} 2$ was common in the SS (46.13\%) compared to the SR (32.26\%, $P=0.004)$. H1 had 0.56 -fold (95\%CI: 0.38-0.84) decreased risk for salt-sensitive hypertension; whereas, $\mathrm{H} 2$ had 1.78-fold (95\%CI: 1.20-2.65) increased risk for saltsensitive hypertension.

\section{Discussion}

In the present study, we firstly performed an association study between PRKG1 gene polymorphism and salt-sensitive hypertension in Chinese. Our result suggested that age and twenty-four hours urinary sodium concentration were the main risk environmental factors associated with salt-sensitive hypertension. Subjects carrying rs7897633-AA, rs1904694-GG genotype and haplotype AG of PRKG1 gene had elevatory risks to be salt-sensitive hypertension, and there were moderate strength interactions between the two SNPs and 24hour urinary sodium concentration associated with salt-sensitivity.

Epidemiology, clinical and experimental researches have showed that dietary sodium and renal sodium are associated with blood pressure. However, the mechanism of salt-sensitive hypertension

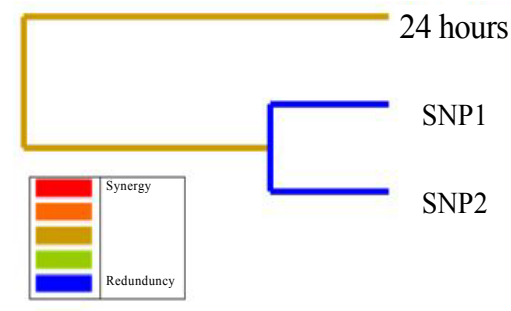

Figure 2: Interaction dendrogram. The different color connections show The degree of interaction from synergy (red) to redundancy (blue). has not been clear so far. About $50 \%$ of essential hypertension patients exists individual heterogeneity of salt-sensitive [15]. Several studies have reported that salt-sensitive hypertension may be related with gender [16], race [17], family history of hypertension [18-20], metabolic syndrome [21] and genetic factors [22]. A dietary interaction study revealed that BP responses to low-sodium and high-sodium interactions were both significantly greater in women compared to men for SBP and DBP [16]. A GenSalt study conducted in rural north China indicated that salt-sensitivity was more common in individuals who were older and had higher basic blood pressure [23]. Similarly, significant differences had been found on changes of SBP between different age groups at low and high sodium feeding periods compared to baseline period, and there were trends of the extent of DBP and MAP increasing with the age at the two periods in the proband who had untreated high normal BP or stage-1 hypertension from 68 families [24]. Our results found there was an association between age and salt-sensitivity, with the increasing of age, the frequencies of SSH significantly increased, which was similar to previous reports.

Many researchers suggested that salt-sensitive hypertension was related to the disordered mechanism of sodium ion and calcium ion transport and the impaired endothelial function [25,26]. After salt load test the inhibition of PRKG1 isoenzyme reduces the biological activity of nitric oxide, regulating contractility of the vascular smooth muscle cells. Perhaps it may increase intracellular free calcium ions concentration or increase sensitivity to calcium ion of the contractile cells in response to elevate blood pressure. In brief, calcium ion plays an important role in controlling vascular tone, so it makes a significant contribution to regulation of systemic blood pressure [11,27]. It is the character of salt-sensitive hypertension that impaired vasodilatation, which is mediated by nitric oxide appears before hypertension. With the increasing of age and blood pressure, the damage will be aggravated further [26]. Thus, PRKG1 gene is regarded as one of the candidate genes on salt-sensitive hypertension.

PRKG1 gene on human chromosome 10 encodes the soluble $c G M P$ dependent protein kinase type $I \alpha \& I \beta$, a nitro-vasodilator effector, which widely distributes in human cells. PRKG1 isoforms $I \alpha$ and $I \beta$ act as key mediators of the nitric oxide/cGMP signalling pathway and are important components of many signal transduction processes in diverse cell types. The PRKG1 proteins play a central role in regulating cardiovascular and neuronal functions in addition to relaxing smooth muscle tone [28,29], preventing platelet aggregation, and modulating cell growth. This gene is most strongly expressed in all types of smooth muscle, platelets, cerebellar Purkinje cells, hippocampal neurons, and the lateral amygdale [30]. Isoforms $I \alpha$ and $I \beta$ have identical $c G M P$ binding and catalytic domains, but differ in their leucine or isoleucine zipper and auto inhibitory sequences, and therefore differ in their dimerization substrates and kinase enzyme activity.

The pathogenic mechanism of PRKG1 gene on salt sensitive hypertension is not clarified. In 2011, Citterio et al. [13] conducted a Genome Wide Association Study which found a strong association with variation in diastolic blood pressure after acute salt loading for a cluster

\begin{tabular}{|c|c|c|c|c|c|c|c|c|c|}
\hline Haplotype & SNP1 & SNP2 & Frequency & SSH N (\%) & SRH N (\%) & $\mathbf{X}^{\mathbf{2}}$ & P & OR & 95\% CI \\
\hline H1 & C & A & 0.469 & $44.4(35.81)$ & $269.1(49.47)$ & 7.54 & $0.006^{*}$ & 0.56 & $0.38-0.84$ \\
\hline H2 & A & G & 0.348 & $57.2(46.13)$ & $175.5(32.26)$ & 8.53 & $0.004^{*}$ & 1.78 \\
\hline H3 & A & A & 0.152 & $17.8(14.35)$ & $83.9(15.42)$ & 0.09 & 0.768 & 0.93 & $0.54-1.61$ \\
\hline H4 & C & G & 0.030 & $4.6(3.71)$ & $15.5(2.85)$ & 0.25 & 0.618 & 1.39 & $0.50-3.87$ \\
\hline
\end{tabular}

Note: $\mathrm{n}(\%)$, numbers and frequencies; ${ }^{*} \mathrm{P}<0.05$. P values were calculated by $\mathrm{X}^{2}$ test; OR, odd ratio; $95 \% \mathrm{Cl}, 95 \%$ confidence interval

Table 4: PRKG1 haplotype frequencies of salt-sensitivity and salt-resistance subjects. 
Citation: Liu Z, Wu J, Zhang J, Liu Y, Liu B, et al. (2014) The Association Study of PRKG1 Gene Polymorphism and Salt-Sensitive Hypertension among the Essential Hypertension in Beijing. J Hypertens 3: 162. doi:10.4172/2167-1095.1000162

Page 6 of 7

of tag-SNPs mapping in the first introns of PRKG1 gene (rs7897633) in Italians. However, it was not demonstrated that whether PRGK1 exists interactions in gene-gene and gene-environment. Yet, we found that there was no significant interaction between the two tag-SNPs and there was moderate strength interaction between the two tag-SNPs with twenty-four hours urinary sodium content related to salt-sensitivity. Studies suggest that a high conservation of the SNP1-A alleles within a moderately conserved flanking intronic region, meaning the ancestral nature of this nucleotide [13]. The SNP1-A allele is a risk gene for saltsensitive response to salt intake, whereas the SNP1-C allele has no significant influence on blood pressure after salt load. In addition, in Hap Map populations the SNP1-A allele is present at high frequency in Sub-Saharan Africans and Kenyans, and at very low frequency in Africans and Chinese who lives in Metropolitan Denver. Meanwhile the SNP2-G allele is present at high frequency in Kenyans and at very low frequency in Chinese who lives in Metropolitan Denver. This study indicated that there were no significant differences at mean arterial pressure variation between different genotypes of SNP1 and SNP2 after acute salt load or furosemide natriuretic test for two hours. Haplotype analysis showed that the frequencies of $\mathrm{H} 1$ and $\mathrm{H} 2$ were higher than other haplotypes in the crowd. Haplotype1 which consists of protection alleles of the two tag-SNPs shares the highest frequency in the salt-resistance group. Conversely, Haplotype 2 which includes risk alleles of the two tag-SNPs shares the highest frequency in the saltsensitivity group. Another study indicates that PRKG1 impinges on circadian rhythms, influencing a mass of physiological processes such as cardiovascular activity [31]

Currently salt sensitivity determination method is mainly either acute or chronic salt load test, but there still remain a lot of limitations. Chronic salt load test is more appropriate to people's eating habits, and is more closed to effect on blood pressure for a long-term salt load in our real life. However, the test period is usually much longer, and cannot avoid the influence of ordinary diet to the consequences during the test. There were more missing participants because they were unable to tolerate the changes in tastes. Therefore, this method is not appropriate for the cohort study. While it does not take a long time for acute salt load test, only a few hours, and it is convenient for quality control [31,32]. In our study, participants received acute salt load test, and it was dangerous to the elderly people. So they needed to be monitored closely. At present, salt sensitivity determination methods are too complicated and troublesome to be appropriate for screening in mass. It is urgently need to find an easier and more acceptable method for salt sensitivity determination in the future.

\section{Conclusion}

Age and twenty-four hour urinary sodium concentration were the environmental risk factors associated with salt-sensitive hypertension. Genotypes of SNP1-AA, SNP2-GG and haplotype AG of PRKG1 gene were risk factors for salt-sensitivity in essential hypertension. There was moderate strength interaction between the two tag-SNPs with twenty-four hours urinary sodium content related with salt-sensitivity.

\section{Acknowledgement}

This work was financially supported by the grants from Natural Science Foundation of China (81373076), Beijing Municipal Commission of Education (SQKM201210025010), and Funding Project for Academic Human Resources Development in Institutions of Higher Learning under the Jurisdiction of Beijing Municipality (PXM2011-014226-07-000028), Beijing Municipal Commission of Education for Overseas Students (2011-No165).

\section{Reference}

1. Michael SK, Surks HK, Wang Y, Zhu Y, Blanton R, et al. (2008) High blood pressure arising from a defect in vascular function. Proc Natl Acad Sci U S A 105: 6702-6707.

2. Liu Zhiquan, Hou Rong (1998) The distribution of salt-sensitive in the population and the character of normotensive among salt-sensitive. Chinese Journal of Hypertension 6: 30-33.

3. Tang Yantian, Wu Qi, Hu Ze, Zhang Shun'an, Yao Xueyan (2012) The analysis of blood pressure variability in salt sensitive hypertensive patients. China Modern Doctor 24: 44-45.

4. Luft FC (2013) Chloride transport and novel insights into salt-sensitive hypertension. J Mol Med (Berl) 91: 539-540.

5. Ando K, Fujita T (2012) Pathophysiology of salt sensitivity hypertension. Ann Med 44 Suppl 1: S119-126.

6. Zhang L, Miyaki K, Wang W, Muramatsu M (2010) CYP3A5 polymorphism and sensitivity of blood pressure to dietary salt in Japanese men. J Hum Hypertens 24: 345-350.

7. Beeks E, Kessels AG, Kroon AA, van der Klauw MM, de Leeuw PW (2004) Genetic predisposition to salt-sensitivity: a systematic review. J Hypertens 22 1243-1249.

8. Kelly TN, He J (2012) Genomic epidemiology of blood pressure salt sensitivity J Hypertens 30: 861-873.

9. Song Y, Miyaki K, Araki J, Zhang L, Takahashi Y, et al. (2008) Influence of CYP11B2 gene polymorphism on the prevalence of hypertension and the blood pressure in Japanese men: interaction with dietary salt intake. J Nutrigenet Nutrigenomics 1: 252-258.

10. Zhang L, Miyaki K, Araki J, Song Y, Kimura T, et al. (2006) Interaction of angiotensin l-converting enzyme insertion-deletion polymorphism and daily salt intake influences hypertension in Japanese men. Hypertens Res 29: 751-758.

11. Lincoln TM, Dey N, Sellak H (2001) Invited review: cGMP-dependent protein kinase signaling mechanisms in smooth muscle: from the regulation of tone to gene expression. J Appl Physiol (1985) 91: 1421-1430.

12. Hofmann F (2005) The biology of cyclic GMP-dependent protein kinases. J Bio Chem 280: 1-4.

13. Citterio L, Simonini M, Zagato L, Salvi E, Delli Carpini S, et al. (2011) Genes involved in vasoconstriction and vasodilation system affect salt-sensitive hypertension. PLoS One 6: e19620.

14. Sullivan JM (1991) Salt sensitivity. Definition, conception, methodology, and long-term issues. Hypertension 17: 161-68.

15. Tiffin N, Meintjes A, Ramesar R, Bajic VB, Rayner B (2010) Computational analysis of candidate disease genes and variants for salt-sensitive hypertension in indigenous Southern Africans. PLoS One 5: e12989.

16. He J, Gu D, Chen J, Jaquish CE, Rao DC, et al. (2009) Gender difference in blood pressure responses to dietary sodium intervention in the GenSalt study. J Hypertens 27: 48-54.

17. Chun TY, Bankir L, Eckert GJ, Bichet DG, Saha C, et al. (2008) Ethnic differences in renal responses to furosemide. Hypertension 52: 241-248.

18. Gu D, Rice T, Wang S, Yang W, Gu C, et al. (2007) Heritability of blood pressure responses to dietary sodium and potassium intake in a Chinese population. Hypertension 50: 116-122.

19. Miller JZ, Weinberger MH, Christian JC, Daugherty SA (1987) Familial resemblance in the blood pressure response to sodium restriction. Am J Epidemiol 126: 822-830.

20. Zhou Liyun, Shen Chong, Yao Cailiang, Gu Dongfeng, Wang Ning (2005) The epidemiology study of blood pressure and salt sensitivity among 253 cases of farmers in northern China. Chinese Journal of Public Health 21: 612-613.

21. Chen J, Gu D, Huang J, Rao DC, Jaquish CE, et al. (2009) Metabolic syndrome and salt sensitivity of blood pressure in non-diabetic people in China: a dietary intervention study. Lancet 373: 829-835.

22. Trudu M, Janas S, Lanzani C, Debaix H, Schaeffer C, et al. (2013) Common noncoding UMOD gene variants induce salt-sensitive hypertension and kidney damage by increasing uromodulin expression. Nat Med 19: 1655-1660. 
Citation: Liu Z, Wu J, Zhang J, Liu Y, Liu B, et al. (2014) The Association Study of PRKG1 Gene Polymorphism and Salt-Sensitive Hypertension among the Essential Hypertension in Beijing. J Hypertens 3: 162. doi:10.4172/2167-1095.1000162

Page 7 of 7

23. Chen J (2010) Sodium sensitivity of blood pressure in Chinese populations. Curr Hypertens Rep 12: 127-134.

24. He guishun (2009) Population study on epidemiology of blood pressure saltsensitivity. Shandong University.

25. Shi Jie, Cao Runlin, Wei Haoguo (2012) Salt sensitive hypertension. Chinese Remedies and Clinics 12: 1172-1173.

26. Mou Jianjun (2011) Progress of Salt in Pathogenesis of Essential Hypertension. Advances in Cardiovascular Diseases 2: 22-25.

27. Hofmann F (2005) The biology of cyclic GMP-dependent protein kinases. J Bio Chem 280: 1-4.

28. Geiselhöringer A, Werner M, Sigl K, Smital P, Wörner R, et al. (2004) IRAG is essential for relaxation of receptor-triggered smooth muscle contraction by cGMP kinase. EMBO J 23: 4222-4231.
29. Surks HK, Mochizuki N, Kasai Y, Georgescu SP, Tang KM, et al. (1999) Regulation of myosin phosphatase by a specific interaction with cGMPdependent protein kinase lalpha. Science 286: 1583-1587.

30. Geiselhöringer A, Gaisa M, Hofmann F, Schlossmann J (2004) Distribution of IRAG and cGKI-isoforms in murine tissues. FEBS Lett 575: 19-22.

31. Feil R, Hölter SM, Weindl K, Wurst W, Langmesser S, et al. (2009) cGMPdependent protein kinase I, the circadian clock, sleep and learning. Commun Integr Biol 2: 298-301.

32. Hou Rong, Liu Zhiquan, Yang Dingyi, Liu Wenhui, Liu Jie (1997) The casecontrol study of acute intravenous salt loading and chronic salt load test in salt sensitivity. Journal of Hypertension 5: 22-24. 